\title{
Improvement of Handoff Latency by GPS based Handoff Technique
}

\author{
Debabrata Sarddar \\ Department of Electronics and \\ Telecommunication Engg, Jadavpur \\ University, \\ Kolkata - 700032
}

Sumon Kr. Bose

Department of Electronics and

Telecommunication Engg,

Jadavpur University, Kolkata 700032

\author{
Shubhajeet Chatterje \\ Department of Electronics and \\ Communication Engg, Institute of Engg. \\ \& Managment college, saltlake, Kolkata- \\ 700091. \\ Shaik Sahil Babu \\ Department of Electronics and \\ Telecommunication Engg, Jadavpur \\ University, Kolkata - 700032
}

Mrinak Kanti Naskar

Department of Electronics and

Telecommunication Engg, Jadavpur

University,

Kolkata - 700032

\author{
Pingakshya Goswami \\ Department of Electronics and \\ Telecommunication Engg, Tezpur \\ University.
}

\section{Utpal Biswas}

Department of Computer Science and Engg, University of Kalyani, Nadia,

West Bengal, Pin- 741235.

\begin{abstract}
Presently IEEE $802.11 \mathrm{~b}$ based wireless networks are being widely used in various fields like personal as well as business applications. Handoff is a critical issue in IEEE 802.11 b based wireless networks. When a mobile node $(\mathrm{MN})$ moves away from the range of its current access point (AP) it needs to perform a link layer handoff. This causes data loss and interruption in communication. According to IEEE 802.11b link layer2 (L2) handoff consists of three phases - scanning, authentication and re-association. Scanning process delay is $90 \%$ of the total handoff delay. So in this paper we propose a better scanning mechanism to reduce handoff latency. Using GPS we determine the direction of velocity of the MN as well as position. It reduces the time to find the closest and best AP among all neighbor APs. This process effectively reduces the handoff latency.
\end{abstract}

\section{Keywords}

IEEE 802.11b, Handoff Latency, GPS (Global Positioning System), Direction of velocity of MN, Neighbor APs, Mobile node $(\mathrm{MN})$.

\section{INTRODUCTION}

Handoff has become an essential criterion in mobile communication system especially in urban areas, owing to the limited coverage area of Access Points (AP). Whenever a MN move from current AP to a new AP it requires handoff. For successful implementation of seamless Voice over IP communications, the handoff latency should not exceed 50ms. But measurements indicate MAC layer handoff latencies in the range of $400 \mathrm{~ms}$ which is completely unacceptable and thus must be reduced for wireless networking to fulfil its potential.
With the advent of real time applications, the latency and packet loss caused by mobility became an important issue in Mobile Networks. The most relevant topic of discussion is to reduce the IEEE 802.11 link-layer handoff latency. IEEE 802.11 MAC specification [1] defines two operation modes: ad hoc and infrastructure mode. In the ad hoc mode, two or more stations (STAs) recognize each other through beacons and hence establish a peer-to-peer relationship. In infrastructure mode, an AP provides network connectivity to its associated STAs to form a Basic Service Set (BSS). Multiple APs form an Extended Service Set (ESS) that constructs the same wireless networks.

\subsection{Channel distribution}

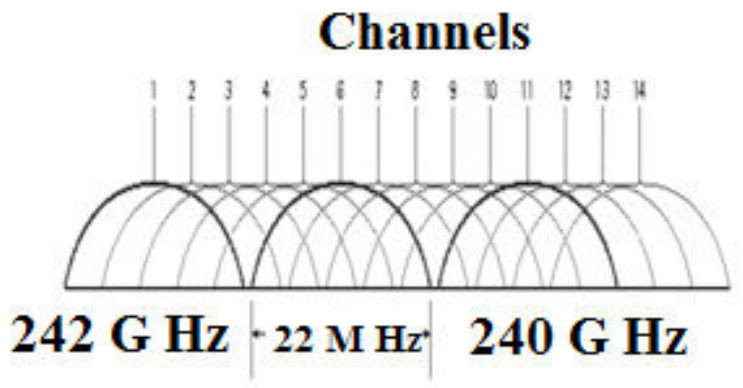

Figure.1

IEEE $802.11 \mathrm{~b}$ and IEEE802.11g operates in the $2.4 \mathrm{GHz}$ ISM band and use 11 of the maximum 14 channels available and are hence compatible due to use of same frequency channels. The channels (numbered 1to14) are spaced by $5 \mathrm{MHz}$ with a bandwidth of $22 \mathrm{MHz}, 11 \mathrm{MHz}$ above and below the centre of the 
channel. In addition there is a guard band of $1 \mathrm{MHz}$ at the base to accommodate out-of-band emissions below $2.4 \mathrm{GHz}$. Thus a transmitter set at channel one transmits signal from $2.401 \mathrm{GHz}$ to $2.423 \mathrm{GHz}$ and so on to give the standard channel frequency distribution as shown in [Figure.1].

It should be noted that due to overlapping of frequencies there can be significant interference between adjacent APs. Thus, in a well configured network, most of the APs will operate on the non-overlapping channels numbered 1, 6 and 11 .

\subsection{Handoff}

When a MN moves out of reach of its current AP it must be reconnected to a new AP to continue its operation. The search for a new AP and subsequent registration under it constitute the handoff process which takes enough time (called handoff latency) to interfere with proper functioning of many applications.

Three strategies have been proposed to detect the need for hand off[2]:

1.2.1 Mobile-controlled-handoff (MCHO): The mobile station(MS) continuously monitors the signals of the surrounding base stations(BS)and initiates the hand off process when some handoff criteria are met.

1.2.2 Network-controlled-handoff (NCHO): The surrounding BSs measure the signal from the $\mathrm{MN}$ and the network initiates the handoff process when some handoff criteria are met.

1.2.3 Mobile-assisted-handoff (MAHO): The network asks the $\mathrm{MN}$ to measure the signal from the surrounding BSs.the network make the handoff decision based on reports from the MS.

Handoff can be of many types:

Hard \& soft handoff: Originally hard handoff was used where a station must break connection with the old AP before joining the new AP thus resulting in large handoff delays. However, in soft handoff the old connection is maintained until a new one is established thus significantly reducing packet loss.

In NGWS(next generation wireless system), two types of handoff scenarios arise: horizontal handoff, vertical handoff[3][4].

Horizontal Handoff: When the handoff occurs between two BSs of the same system it is termed as horizontal handoff. It can be further classified into two:

Link layer handoff : Horizontal handoff between two BSs that are under the same foreign agent(FA).

Intra system handoff : Horizontal handoff between two BSs that belong to two different FAs and both FAs belong to the same gateway foreign agent (GFA) and hence to the same system.

Vertical Handoff : When the handoff occurs between two BSs that belong to two different GFAs and hence to two different systems it is termed as vertical handoff as shown in figure 3.

The handoff procedure consists of three logical phases where all communication between the mobile station undergoing handoff and the APs concerned is controlled by the use of IEEE802.11 management frames as shown below in [fig2].

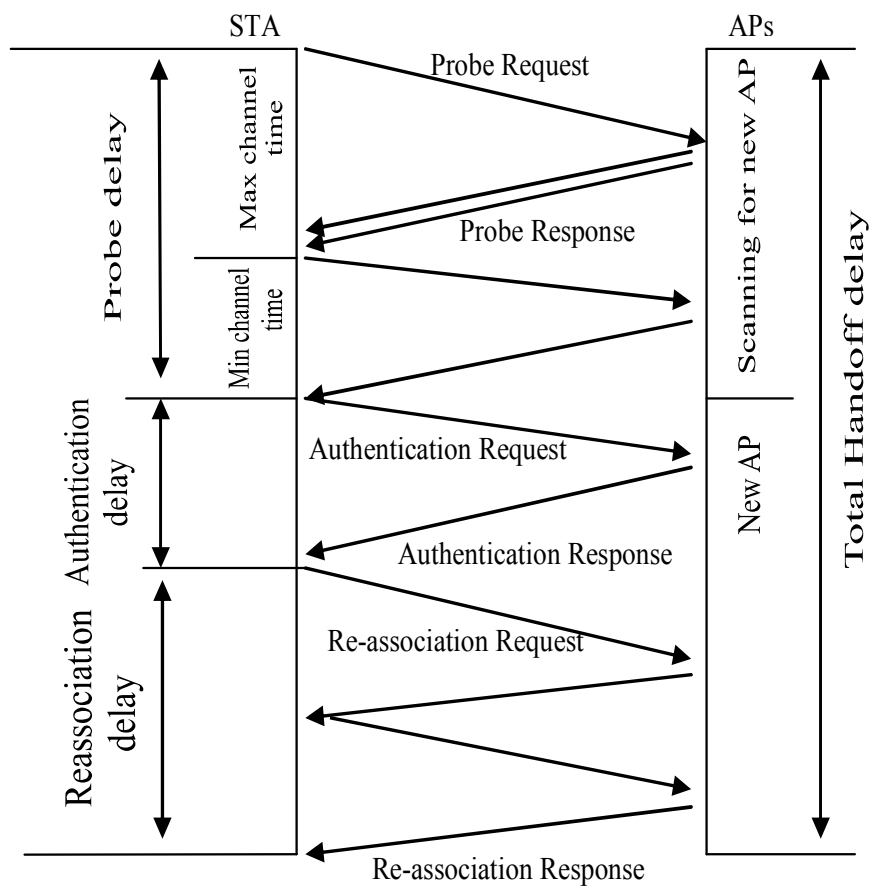

Figure.2

Scanning: When a mobile station is moving away from its current AP, it initiates the handoff process when the received signal strength and the signal-to-noise-ratio have decreased significantly. The STA now begins MAC layer scanning to find new APs. It can either opt for a passive scan (where it listens for beacon frames periodically sent out by APs) or chose a faster active scanning mechanism wherein it regularly sends out probe request frames and waits for responses for $\mathrm{T}_{\mathrm{MIN}}$ (min Channel Time) and continues scanning until $\mathrm{T}_{\mathrm{MAX}}$ (max Channel Time) if at least one response has been heard within $\mathrm{T}_{\text {MIN }}$. Thus, $\mathrm{n}^{*} \mathrm{~T}_{\text {MIN }}$ $\leq$ time to scan $n$ channels $\leq \mathrm{n}^{*} \mathrm{~T}_{\mathrm{MAX}}$. The information gathered is then processed so that the STA can decide which AP to join next. The total time required until this point constitutes $90 \%$ of the handoff delay.

Authentication: Authentication is necessary to associate the link with the new AP. Authentication must either immediately proceed to association or must immediately follow a channel scan cycle. In pre-authentication schemes, the MN authenticates with the new AP immediately after the scan cycle finishes. IEEE 802.11 defines two subtypes of authentication service: 'Open System' which is a null authentication algorithm and 'Shared Key' which is a four-way authentication mechanism. If Inter Access Point Protocol (IAPP) is used, only null authentication frames need to be exchanged in the re-authentication phase. Exchanging null authentication frames takes about 1-2 ms.

Re-Association: Re-association is a process for transferring associations from old AP to new one. Once the STA has been authenticated with the new AP, re-association can be started. Previous works has shown re-association delay to be around 1-2 ms. The range of scanning delay is given by:- 
$\mathrm{N} \times$ Tmin _ Tscan _ $\mathrm{N} \times$ Tmax

Where $\mathrm{N}$ is the total number of channels according to the spectrum released by a country, Tmin is Min Channel Time,Tscan is the total measured scanning delay, and Tmax is Max Channel Time. Here we focus on reducing the scanning delay by minimizing the total number of scans performed. This paper is organized as follows. First we present a brief overview of IEEE $802.11 \mathrm{~b}$ standard handoff procedure, GPS in handoff management and related work in Section-2. In Section-3 the proposed work is presented. Finally in Section- 4 we demonstrate simulation results and in Section-5 a brief conclusion.

\section{RELATED WORK}

A number of different schemes have been proposed to reduce handoff latency in IEEE 802.11 wireless LANs. IEEE 802.11b based wireless and mobile networks [5], also called Wi-Fi commercially, are experiencing a very fast growth upsurge and are being widely deployed for providing variety of services as it is cheap, and allows anytime, anywhere access to network data. The new age applications require a seamless handover while the small coverage of individual APs has increased the number of handoffs taking place. Thus reducing the handoff latency has become a burning issue and much work has been done to achieve this. See [6] for an overall review of popular methods suggested.

Shin et al in [7] have introduced a selective scanning algorithm with the help of channel masking technique coupled with a caching mechanism to significantly reduce the handoff delay. However, it still scans excess APs even after the new AP may have already been found and thus leaves room for further improvements.

Handoff, an inherent problem with wireless networks, particularly real time applications, has not been well addressed in IEEE 802.11, which takes a hard handoff approach [8].

In [9] the authors have introduced a novel caching process using neighbor graphs by pre-scanning neighbor APs to collect their respective channel information. The concept of neighbor graphs can be utilized in different ways and have become very popular in this field. In [10] a pre-authentication mechanism is introduced to facilitate seamless handover. [11] is a novel approach towards reducing handover latency in AP dense networks.

Besides, much progress has been made in introducing GPS aided handoffs; vide [12] to [13]. To reduce handoff latency in wireless LAN using IAPP [14], an algorithm on context transfer mechanism using 'Neighbor Graph' (NG) [15] was suggested in [16]. However, IAPP was only reactive in nature and creates an additional delay in a handoff. One approach on Physical layer (PHY) is the method using two trans-receivers, where a wireless mobile node (MN) has two Wireless Network Interface Cards (WNICs) [17], one for keeping connection to current AP and the other for scanning channels to search for alternate APs [18].

In our work also we look at another such position dependant solution with a view to minimize overhead signalling problems. This is necessary since extensive pre-scanning is unacceptable in high traffic AP dense networks.

\section{PROPOSED WORKS}

We consider a hexagonal cell is surrounded by six other hexagonal cells ( 7 cell cluster). During the movement of the mobile node $(\mathrm{MN})$ it can move to any one of the two adjacent APs. As shown in figure 3 the MN can go to either AP1 or AP2.
We take a hexagonal cell (coverage area of old AP) and divide it into three regions as shown in figure5. We define (i) $\mathrm{P}_{1} \mathrm{P}_{2} \mathrm{P}_{3} \mathrm{P}_{4} \mathrm{P}_{5} \mathrm{P}_{6} \mathrm{P}_{1}$ as core area where the $\mathrm{MN}$ does not need handoff.(ii) $\Delta \mathrm{A}_{\mathrm{i}} \mathrm{P}_{\mathrm{i}+1} \mathrm{~A}_{\mathrm{i}+1}\left(\mathrm{i}=1\right.$ to 5 ) and $\Delta \mathrm{A}_{1} \mathrm{P}_{1} \mathrm{~A}_{6}$ as $\Delta_{1}$ region (overlap region) where $\mathrm{MN}$ already finds its best AP using GPS. And (iii) $\Delta \mathrm{P}_{\mathrm{i}} \mathrm{A}_{\mathrm{i}} \mathrm{P}_{\mathrm{i}+1}$ (i=1 to 5) and $\Delta \mathrm{P}_{6} \mathrm{~A}_{6} \mathrm{P}_{1}$ as $\Delta_{2}$ region.

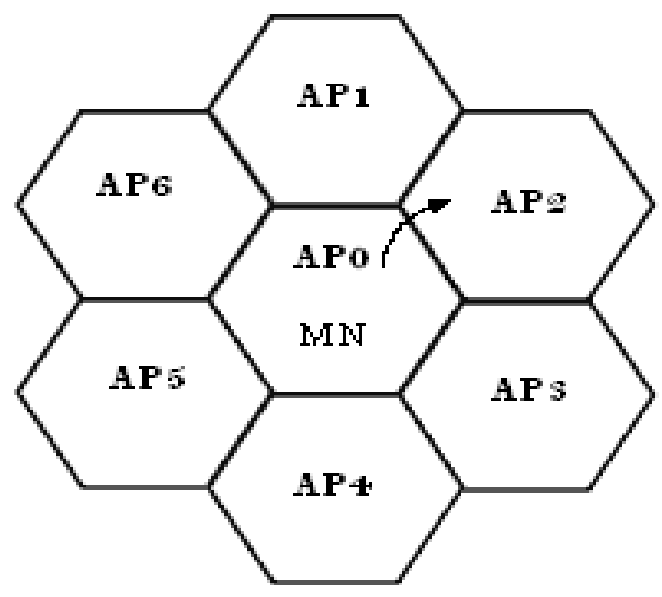

Figure3 cell cluster

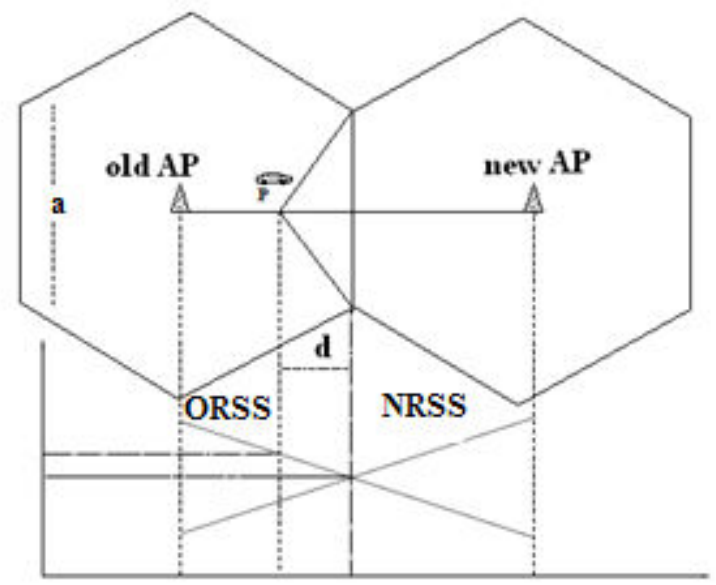

Figure 4

If the $\mathrm{MN}$ is in one of the $\Delta_{2}$ regions then there is a possibility of handoff. In these regions handoff may occur between old AP (AP0) and any one of two adjacent APs. In the above figure handoff may occur between AP0 and AP1 or between AP0 and AP2.

To decide where the mobile node (MN) moves (one of two APs for handoff) we depend on the direction of velocity of the MN. The decision making procedure will be as follows.

(i)First to determine the MN's current position i.e. the $\mathrm{MN}$ is in $\Delta_{2}$ region or not.

(ii) Determine direction of velocity of the $\mathrm{MN}$.

(iii) Total no of data to be taken into account. 


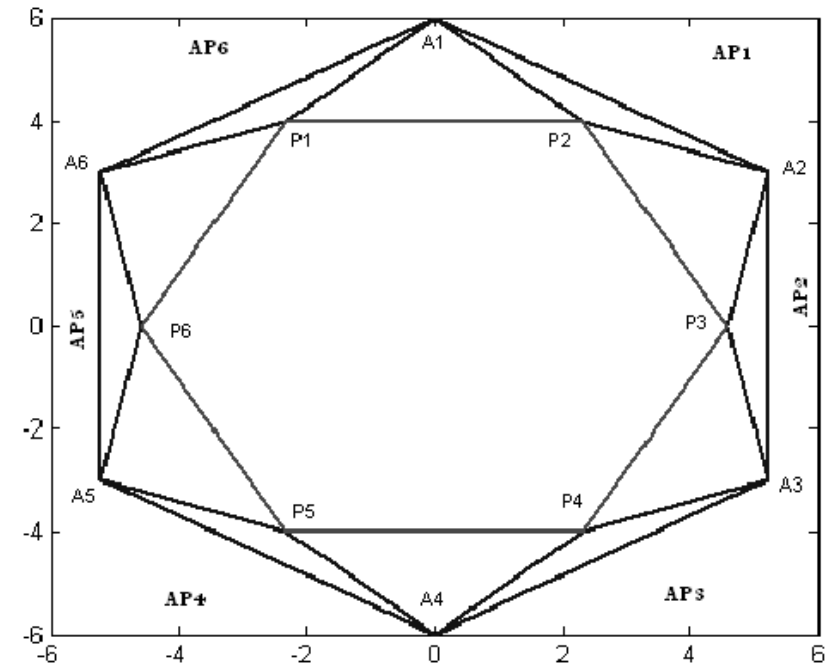

Figure 5

For our work we consider that the $\mathrm{MN}$ is now in $\Delta \mathrm{P}_{2} \mathrm{~A}_{2} \mathrm{P}_{3}$ region (Figure5). To confirm, it finds the equation of straight line $\mathrm{P}_{2} \mathrm{P}_{3}$. The coordinates of $\mathrm{P}_{2}$ and $\mathrm{P}_{3}$ are $[(\sqrt{3} \mathrm{a} / 2-\mathrm{d}) / 2,(\sqrt{3} \mathrm{a} / 2-$ d) $\sqrt{ } 3 / 2]$ and $[(\sqrt{3} a / 2-d), 0]$ respectively. So the equation is

$$
\begin{gathered}
a 1 x+b 1 y+c 1=0 \ldots \ldots \ldots(1) \\
{[a 1=\sqrt{ } 3 ; b 1=1 ; c 1=\sqrt{ } 3(d-\sqrt{ } 3 a / 2)]}
\end{gathered}
$$

By GPS, we get the location of MN. So we can determine whether the $\mathrm{MN}$ is out of that region or in that region.

As we find the location of $\mathrm{MN}$ we now determine the direction of velocity. Let at some instant of time the location of $\mathrm{MN}$ is (xi, yi) then the angle (with $\mathrm{A}_{2} \mathrm{P}_{3}$ ) is

$$
\begin{aligned}
& \mathrm{mi}=\arctan {[(\mathrm{A} 2 \mathrm{~B} 1-\mathrm{A} 1 \mathrm{~B} 2) /(\mathrm{A} 1 \mathrm{~A} 2+\mathrm{B} 1 \mathrm{~B} 2)]----(2) . } \\
& \text { where, } \quad \mathrm{A} 1=(\mathrm{a} / 2-\mathrm{yi}) \\
& \mathrm{B} 1=(\mathrm{xi}-\sqrt{3} \mathrm{a} / 2) \\
& \mathrm{A} 2=\mathrm{yi} \\
& \mathrm{B} 2=(\sqrt{3} \mathrm{a} / 2-\mathrm{d}-\mathrm{xi})
\end{aligned}
$$

So the angle mi can be calculated.

So for $i=1,2,3, \ldots \ldots$ we get corresponding angles. If $\mathrm{m} 1<\mathrm{m} 2<\mathrm{m} 3<\ldots$. we can conclude that the $\mathrm{MN}$ is going towards AP2 (Fig6). If $\mathrm{m} 1>\mathrm{m} 2>\mathrm{m} 3>\ldots \ldots$ we can conclude that the $\mathrm{MN}$ is going towards AP1.

Let the average velocity of the MN is $v$ and the maximum scan time is . The MN has to take right decision before it goes to a threshold region whose distance is from the cell boundary. Where

Let the distance between the MN and the threshold region is $d$ and the MN takes $t$ to reach the threshold region. Therefore $t$ $=d / v$. If the GPS information is sent at an interval of, then total no of received data is $n=t$ /

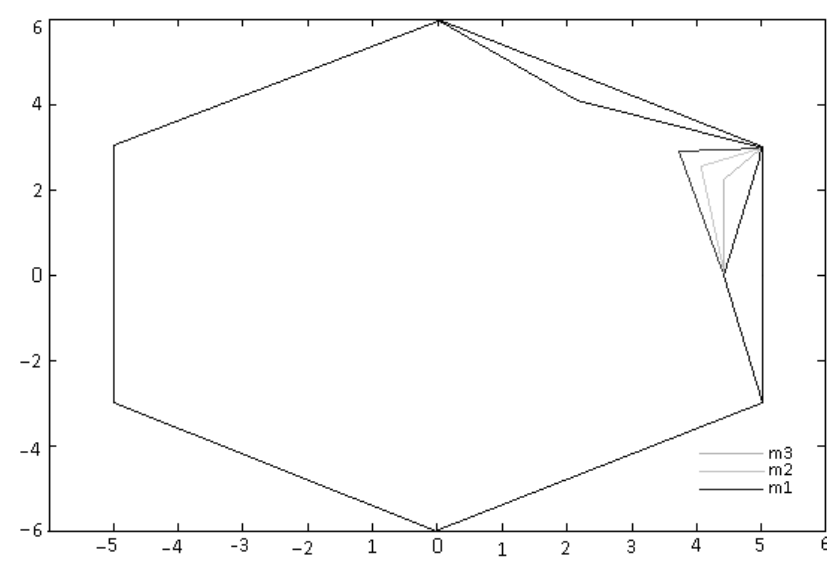

Figure 6

\subsection{Handoff Management}

The GPS server chooses the new target AP from these two adjacent APs. The server sends a handoff initiate (HI) message to the MN and provides parameters like the target AP's IEEE $802.11 \mathrm{~b}$ channels, SSID (Service Set Identifier).

Getting the $\mathrm{HI}$ message the MN starts handover process and tries to associate to the target AP using the relevant information sent by the GPS server. As the MN already knows its destination, it only sends a probe request message and waits for the target AP's specific probe response. Receiving the probe response the MN starts authentication and then re-association process. Therefore, the delay for scanning process is greatly reduced. Preregistration process significantly reduces the link layer2 handoff latency.

\subsection{Proposed algorithm}

To apply this method in real field, we follow the following algorithm.

*/ We consider the cell is divided in three regions as shown in figure 5. (i) core area ii) $\Delta_{1}$ region (overlap region) And (iii) $\Delta_{2}$ region.*/

*/the position of MN at $t$ instant measured by GPS is given as $(\mathrm{xt}, \mathrm{yt}) * /$

*/ Size of each side of cell is a*/

1. Find the equation of the straight line $P_{i} P_{i+1}$

$$
\mathrm{X}_{\mathrm{i}} \mathrm{x}+\mathrm{Y}_{\mathrm{i}} \mathrm{y}+\mathrm{Z}_{\mathrm{i}}=0
$$

2. Calculate the constant $\mathrm{K}$.

$\mathrm{K}=\mathrm{X}_{\mathrm{i}} \mathrm{x}_{\mathrm{t}}+\mathrm{Y}_{\mathrm{i}} \mathrm{y}_{\mathrm{t}}+\mathrm{Z}_{\mathrm{i}}$

3. $\mathrm{i}=1$.

4. while $(\mathrm{i}=<5)$

4.1. If $K=Z_{i}$

4.1.1. handoff is not required. $* / \mathrm{MN}$ and origin is at the same side of straight line */

4.2. If $K=-Z_{i}$ 
4.2.1. handoff is required in $* / \mathrm{MN}$ and origin is at the opposite side straight line */

\subsection{Exit.}

4.4. End.

4.5. We will calculate the angle of the location of MN (xt,yt) with the straight line $\mathrm{A}_{\mathrm{i}} \mathrm{P}_{\mathrm{i}+1}$.

$m j=\arctan \left[\left(\mathrm{A}_{\mathrm{i}} \mathrm{B}_{\mathrm{i}-1}-\mathrm{A}_{\mathrm{i}-1} \mathrm{~B}_{\mathrm{i}}\right) /\left(\mathrm{A}_{\mathrm{i}-1} \mathrm{~A}_{\mathrm{i}}+\mathrm{B}_{\mathrm{i}-1} \mathrm{~B}_{\mathrm{i}}\right)\right] \quad$ where,

$\mathrm{A}_{\mathrm{i}-}$

${ }_{1}=(\mathrm{a} / 2-\mathrm{yi}), \mathrm{B}_{\mathrm{i}-1}=(\mathrm{xi}-\sqrt{3} \mathrm{a} / 2), \mathrm{A}_{\mathrm{i}}=\mathrm{yi}, \mathrm{B}_{\mathrm{i}}=(\sqrt{3} \mathrm{a} / 2-\mathrm{d}-\mathrm{xi})$

4.6. while $(j=<5)$

4.6.1 If $m j>=m(j+1)$

4.6.2. Exit.

4.6.3. End.

4.7. handoff will be performed at $\mathrm{AP}(\mathrm{j}+1)$.

4.8. while $(\mathrm{j}=<5)$

4.8.1. If $m j=<m(j+1)$

4.8.2. Exit.

4.8.3. End.

4.9. handoff will be performed at APj.

4.10. END.

\section{SIMULATION RESULT}

For our simulation, we consider a macro-cellular system with a cell size of $\mathrm{a}=1 \mathrm{~km}$ and a macro-reference distance $\mathrm{d}=100 \mathrm{~m}$. Let us assume that the co-ordinates of the $\mathrm{MN}$ at $\mathrm{t} 1, \mathrm{t} 2, \mathrm{t} 3$, $\mathrm{t} 4$ and $\mathrm{t} 5$ are $(2.8,3.5),(3,3),(3.5,2.8),(4,2.5)$ and $(4.5,2)$ respectively.

$\mathrm{mi}=\arctan [(\mathrm{A} 2 \mathrm{~B} 1-\mathrm{A} 1 \mathrm{~B} 2) /(\mathrm{A} 1 \mathrm{~A} 2+\mathrm{B} 1 \mathrm{~B} 2)]-----(3)$.

where, $\quad \mathrm{A} 1=(\mathrm{a} / 2-\mathrm{yi})$

$$
B 1=(x i-\sqrt{3} a / 2)
$$

$\mathrm{A} 2=\mathrm{yi}$

$B 2=(\sqrt{ } 3 a / 2-d-x i)$

So the angle mi can be calculated From equation (3) .

For t1 $(2.8,3.5)$,

$\mathrm{xi}=2.8$ and $\mathrm{yi}=3.5$.

$\mathrm{A} 1=(.5-3.5)=-.3$

$\mathrm{B} 1=(2.8-0.87)=1.93$

$\mathrm{A} 2=3.5$

$\mathrm{B} 2=(.87-.1-2.8)=-2.03$

$\mathrm{mi}=\arctan \{(3.5 * 1.93-(-.3)(-2.03)) /(-.3) * 3.5+(-2.03) * 1.93\}$

$\mathrm{mi}=\arctan (6.146 / 4.0229)$

$\mathrm{mi}=56.79$
Similarly we get the angles (in degree) $61.63,74.83,98.36$ and 140.82 respectively. As $[56.79<61.63<74.83<98.36<140.82$ (shown in Fig 7)] we conclude that the direction of velocity of the MN is towards AP2 (Fig 8). Therefore the MN scans the AP2 only.

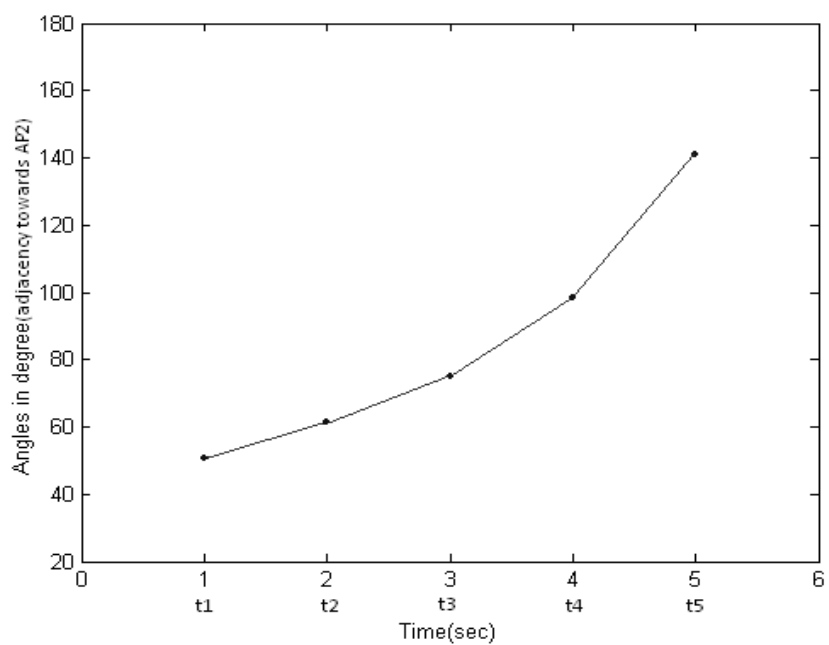

Figure7

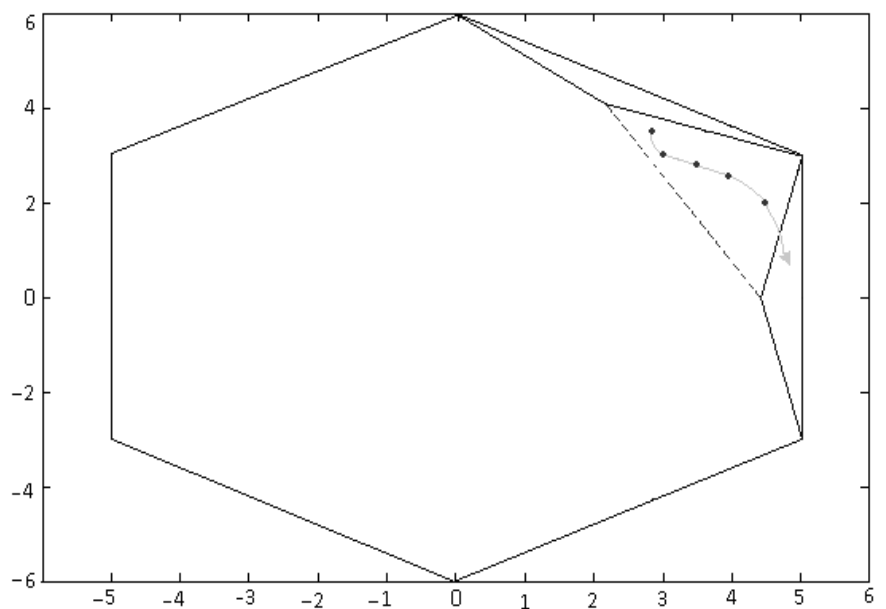

Figure 8

For another case, we assume that the location of the $\mathrm{MN}$ at $\mathrm{t} 1$, $\mathrm{t} 2, \mathrm{t} 3, \mathrm{t} 4$ and $\mathrm{t} 5$ are $(4.2,1),(3.8,1.5),(3.5,2),(3,3),(3.5,3.2)$ respectively.

$\mathrm{mi}=\arctan [(\mathrm{A} 2 \mathrm{~B} 1-\mathrm{A} 1 \mathrm{~B} 2) /(\mathrm{A} 1 \mathrm{~A} 2+\mathrm{B} 1 \mathrm{~B} 2)]-----(2)$.

where, $\quad \mathrm{A} 1=(\mathrm{a} / 2-\mathrm{yi})$

$$
\begin{aligned}
& B 1=(x i-\sqrt{3} a / 2) \\
& A 2=y i \\
& B 2=(\sqrt{3} a / 2-d-x i)
\end{aligned}
$$

So the angle mi can be calculated From equation (2) as before. For point $\mathrm{t} 1(4.2,1)$

$\mathrm{A} 1=(.5-1)=-.5$
$\mathrm{~B} 1=(4.2-.5 * \sqrt{ } 3)=3.33$ 
$\mathrm{A} 2=1$

$\mathrm{B} 2=(\sqrt{ } 3 * .5-.1-4.2)=-3.43$

$m i=\arctan (-1.1809)$

$\mathrm{mi}=130.2578$

Similarly, We calculate the angle (in degree) 107.91, 90.93, 61.63 and 64.07 respectively. As [130.26>107.91>90.93>61.63 $<64.07$ (shown in Fig 9)] the angles are decreasing for most cases the direction of MN's velocity is towards AP1 (Fig.10). The MN scans AP1 only.

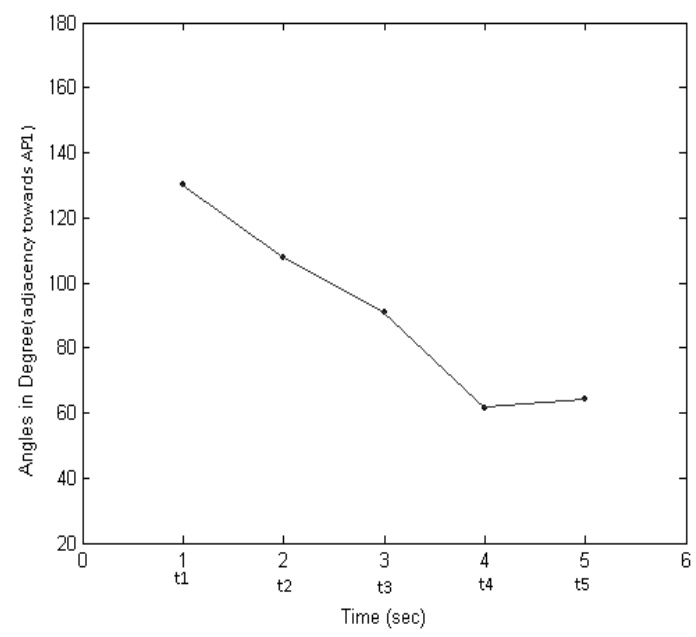

Figure 9.

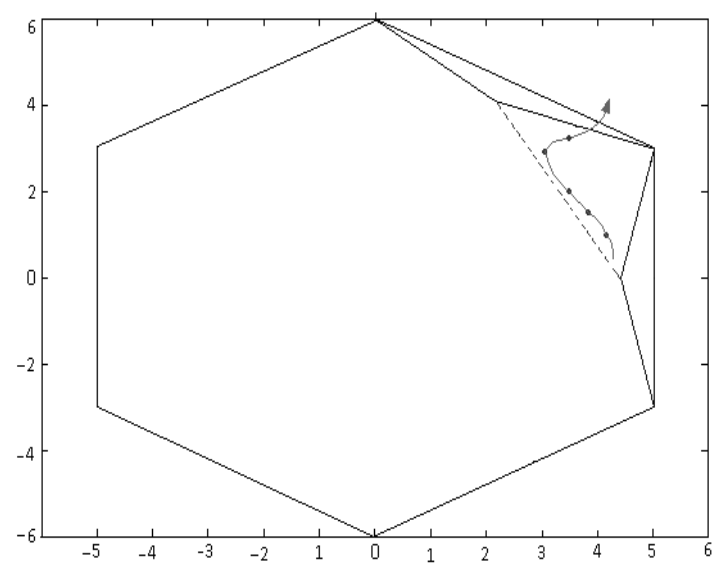

Figure10.

So in this process, the MN does not need to scan all the APs in its vicinity. It scans only one AP to which its adjacency is maximum. Therefore, delay incurred during scanning phase is effectively reduced.

We consider few instances when the randomly generated $\mathrm{MN}$ needs handoff. Different parameters like traffic load at that moment in the network, channels used the AP and available free channels, load on non-overlapping channels etc are taken into our consideration. The MN first looks for the potential AP and then first scans the non-overlapping channels $(1,6,11)$ if present.
If it fails it scans the other channels. We neglect authentication delay as we use pre-authentication during scanning phase. We consider roundtrip time as $3 \mathrm{~ms}$ which is the sum of message processing time and re-association time. We made 100 sample tests run at a regular interval of time to measure handoff delays. Simulation result (Fig 11.) shows that our proposed method reduces the handoff latency to a minimum of around $6 \mathrm{~ms}$ which is much lesser than previous results.

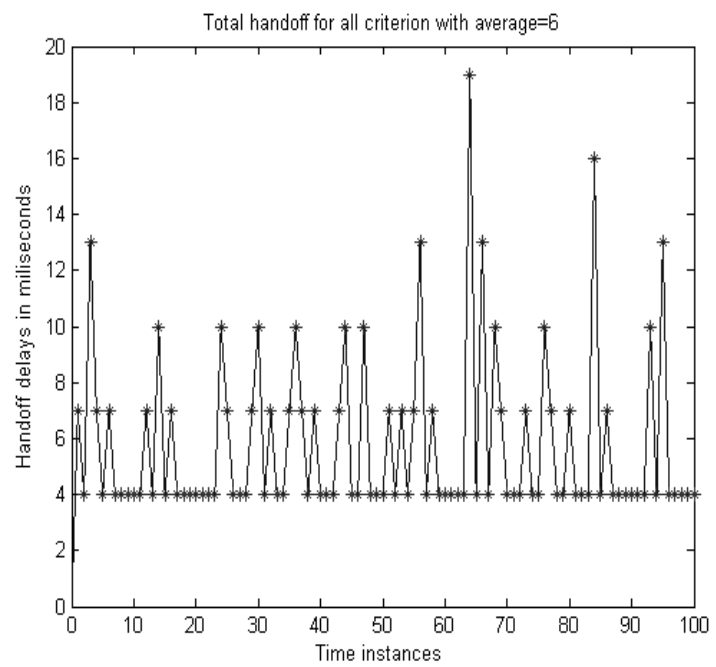

Fig11. Handoff latency measured at different instances

Here we calculate the handoff delay at different time intervals. The different values of handoff latency arise due to traffic problem and different number of free available channels in neighbor APs. But, to find the effectiveness of our proposed algorithm we have to consider the average value. We can see the average handoff latency is $6 \mathrm{~ms}$ which is a significant improvement.

\section{CONCLUSION}

In this paper, we described a GPS based approach that supports mobility in IEEE $802.11 \mathrm{~b}$ WLANs. We proposed a faster handoff mechanism by determining direction of velocity of the MN (using GPS). The location and angle information are stored in the GPS server memory and compared for a specific time interval. It helps us to detect the trajectory through which the $\mathrm{MN}$ is moving towards an AP. Simulation result indicates that our approach considerably reduces the scanning delay. As scanning delay contributes $90 \%$ of the total delay this method reduces the handoff latency drastically.

However, our proposed method leaves some drawbacks which we can leave for the future works.

For example, though we have been able to reduce handoff latencies we do not consider whether the handoff was at all necessary, i.e. ping-pong effects can significantly increase the number of false handoffs taking place. The ping pong condition arises when a MN moves back and forth between two BSs very frequently. Handoff cannot take place in this condition due to this frequent movement of MN. To avoid such problem, one traditional way is that the $\mathrm{MN}$ is allowed to continue 
maintaining a radio link with the old AP until the signal strength from new AP exceeds that of the old AP. But in our proposed method $\mathrm{MN}$ is bound to perform handoff in handoff region i.e. $\Delta_{1}$ region and it has to reject its radio link with the old AP and has to connect with the new AP within that old cell. Thus MN cannot continue its radio link with the old AP any more. So in our proposed mechanism no such cure is possible for ping pong effect. Also, the use of GPS is not an economy friendly approach.

It is worth mentioning here that although the proposed work has been presented considering honeycomb structures yet our algorithm would work in a similar manner for other cell structures and neighbor AP locations. Minor changes would be introduced depending on the network topology.

\section{FUTURE WORKS}

Limitations given in above section may be effectively eliminated by using following techniques. The ping pong effect may be minimized by using received signal strength method along with our proposed algorithm. We can avoid the use of GPS by using neighbor graph or some other coordinate evaluation technique to make our proposed method more economy friendly. We intend to take up these matters in future studies. The real challenge as of now is to interpret the coverage areas of APs geometrically and incorporate that knowledge locally to optimize handoff performances.

\section{REFERENCES}

[1] Hye-Soo Kim, Sang Hee Park, Chun-Su Park, Jae Won Kim and Sung-Jea Ko. "Selective Channel Scanning for Fast Handoff in Wireless LAN using Neighbor Graph", July 2004.

[2] Hongqiang Zhai, Xiang Chen, and Yuguang Fang. "How well can the IEEE 802.11 wireless lan support quality of service?" IEEE Transactions on Wireless Communications, 4(6):3084-3094, December 2005.

[3] Yi-Bing Lin Imrich Chalmatc, "Wireless and Mobile Network Architectures," pp. 17.

[4] AKYILDIZ, I. F., XIE, J., and MOHANTY, S., "A survey on mobility management in next generation all-IP based wireless systems," IEEE Wireless Communications, vol. 11, no. 4, pp. 16-28, 2004.

[5] STEMM, M. and KATZ, R. H., "Vertical handoffs in wireless overlay networks," ACM/Springer Journal of Mobile Networks and Applications(MONET), vol. 3, no. 4, pp. 335-350, 1998.

[6] Jaeyoung Choi Student Member, IEEE, Taekyoung Kwon \& Yanghee Choi, Senior Member IEEE, Sangheon Pack Member, , IEEE,Fast Handoff Support in IEEE 802.11 Wireless Networks.

[7] Arunesh Mishra, Minho Shin \& William Arbaugh, An Empirical Analysis of the IEEE 802.11 MAC Layer Handoff Process. [Anshuman Singh Rawat \& Henning Schulzrinne Reducing MAC Layer Handoff Latency in IEEE 802.11 Wireless LANs.

[8] Sangho Shin, Andrea G. Forte, Context Caching using Neighbor Graphs for Fast Handoffs in a Wireless Network.
[9] S. Park and Y. Choi Pre-authenticated fast handoff in a public wireless LAN based on IEEE802.1x mode IFIP TC6 Personal Wireless Communications. Singapore, October 2002 .

[10] Jin Teng, Changqing Xu, Weijia Jia, Dong Xuan, D-scan: Enabling Fast and Smooth Handoffs in AP-dense802.11 Wireless Networks.

[11] Chien-Chao Tseng, K-H Chi, M-D Hsieh \& H-H Chang, Location-based Fast Handoff for 802.11 Networks. IEEE COMMUNICATIONS LETTERS, VOL9, NO 4 April 2005.

[12] S.Kyriazakos, D. Drakoulis, G.Karetsos, Optimization of the Handover Algorithm based on the Position of the Mobile Terminals. Proceedings of Symposium on Communications and Vehicular Technology, October 2000.

[13] In-Su Yoon, Sang-Hwa Chung, and Tae-Hoon Kim, A fast handover method for IEEE802.11 wireless networks using combined GPS and SNR

[14] J. Pesola \& S. Pokanen, Location-aided Handover in Heterogeneous Wireless Networks. in Proceedings of Mobile Location Workshop, May2003.

[15] Jan Eric Hakegard, Multi-Cell WLAN Coverage and Capacity.

[16] Ping-Jung Huang, Yu-Chee Tseng. "A Fast Handoff Mechanism for IEEE 802.11 and IAPP Networks".

[17] M.Ohta, "Smooth Handover over IEEE 802.11 Wireless LAN, "Internet Draft : draft-ohta- smooth- handover-wlan00.txt, Jun. 2002.

[18] Yogesh Ashok Powar and Varsha Apte, "Improving the IEEE 802.11 MAC Layer Handoff Latency to Support Multimedia Traffic".

\section{Author Profile:}

Debabrata Sarddar is currently pursuing his $\mathrm{PhD}$ at Jadavpur University. He completed his M.Tech in Computer Science \& Engineering from DAVV, Indore in 2006, and his B.Tech in Computer Science \& Engineering from Regional Engineering College (NIT), Durgapur in 2001. His research interest includes wireless and mobile system

Shubhajeet Chatterjee is presently pursuing B.Tech Degree in Electronics and Communication Engg. at Institute of Engg. \& Managment College, under West Bengal University of Technology. His research interest includes wireless sensor networks and wireless communication systems.

SHAIK SAHIL BABU is pursuing Ph.D in the Department of Electronics and Telecommunication Engineering under the supervision of Prof. M.K. NASKAR at Jadavpur University, KOLKATA. He did his Bachelor of Engineering in Electronics and Telecommunication Engineering from Muffa Kham Jah College of Engineering and Technology, Osmania University, Hyderabad, and Master of Engineering in Computer Science and Engineering from Thapar Institute of Engineering and Technology, Patiala, in Collaboration with National Institute of Technical Teachers' Training and Research, Chandigarh. 
Sumon Kumar Bose is presently pursuing B.Tech Degree in Electronics and Communication Engg. at Jadavpur University. His research interest includes wireless sensor networks and wireless communication systems.

Pingakshya Goswami is presently pursuing B.Tech Degree in Electronics and Communication Engg. at school of engineering, under Tezpur University. His research interest includes wireless sensor networks and wireless communication systems

Utpal Biswas received his B.E, M.E and $\mathrm{PhD}$ degrees in Computer Science and Engineering from Jadavpur University, India in 1993, 2001 and 2008 respectively. He served as a faculty member in NIT, Durgapur, India in the department of Computer Science and Engineering from 1994 to 2001. Currently, he is working as an associate professor in the department of Computer Science and Engineering, University of Kalyani, West Bengal, India. He is a co-author of about 35 research articles in different journals, book chapters and conferences. His research interests include optical communication, ad-hoc and mobile communication, semantic web services, E- governance etc.
Mrinal Kanti Naskar received his B.Tech. (Hons) and M.Tech degrees from E\&ECE Department, IIT Kharagpur, India in 1987 and 1989 respectively and Ph.D. from Jadavpur University, India in 2006.. He served as a faculty member in NIT, Jamshedpur and NIT, Durgapur during 1991-1996 and 19961999 respectively. Currently, he is a professor in the Department of Electronics and Tele-Communication Engineering, Jadavpur University, Kolkata, India where he is in charge of the Advanced Digital and Embedded Systems Lab. His research interests include ad-hoc networks, optical networks, wireless sensor networks, wireless and mobile networks and embedded systeMN. He is an author/co-author of the several published/accepted articles in WDM optical networking field that include "Adaptive Dynamic Wavelength Routing for WDM Optical Networks" [WOCN,2006], "A Heuristic Solution to SADM minimization for Static Traffic Grooming in WDM unidirectional Ring Networks" [Photonic Network Communication, 2006], "Genetic Evolutionary Approach for Static Traffic Grooming to SONET over WDM Optical Networks" [Computer Communication, Elsevier, 2007], and "Genetic Evolutionary Algorithm for Optimal Allocation of Wavelength Converters in WDM Optical Networks" [Photonic Network Communications,2008]. 